\title{
Implicaciones teórico-conceptuales en la reelaboración del término comumidad para la praxis social comunitaria
}

Conceptual theoretical implications in the reprocessing of the term community for the communitysocial praxis

\author{
Susana Aurora Burciaga Jiménez \\ María del Rocío Guzmán Benavente \\ Unversidad Juárez del Estado de Durango
}

\section{Resumen}

En el presente estudio reflexionamos acerca de las implicaciones del concepto de comunidad desde un ejercicio de praxis social y, específicamente, desde la Psicología Social Comunitaria (PSC). Plantamos una diferencia sustancial entre el enfoque positivista y la visión liberadora de la PSC a través del mismo concepto y retomamos algunos aspectos contemplados en las propuestas de diversos autores sobre los elementos que éste contiene; en dicho contexto, analizamos su congruencia de acuerdo a lo que entendemos por praxis social comunitaria. Asimismo, nos interrogamos acerca del papel de ciertas instancias que pueden estar involucradas en el trabajo de una praxis genuina, al señalar la relación entre la postura de las y los facilitadores comunitarios en relación con las ideas que pueden construir en torno al concepto de comunidad. Finalmente, planteamos la necesidad de trabajar desde cualquier disciplina de estudio social, incluyendo la PSC, en la reelaboración de aquellas certidumbres para evitar caer en relaciones de poder asimétricas con las y los participantes en proyectos comunitarios.

Palabras Clave: Psicología Social Comunitaria (PSC), praxis social, praxis social comunitaria, comunidad.

Nota de Autor:

Susana Aurora Burciaga Jiménez, Departamento de Psicología, Universidad Juárez del Estado de Durango; María del Rocío Guzmán Benavente, Departamento de Psicología, Universidad Juárez del Estado de Durango.

La correspondencia en relación con este artículo debe dirigirse a Susana Aurora Burciaga Jiménez, Departamento de Psicología, Universidad Juárez del Estado de Durango, Constitución 404 sur, Zona centro, C.P. 34000, Durango, Durango, México.

Correo electrónico: susanabj@ hotmail.com 


\begin{abstract}
We reflect on the implications of the concept of community from an exercise of social praxis and specifically from the Social Community Psychology (SCP). We planted a substantial difference between the positivist approach and vision SCP releasing through the same concept, retaking certain aspects across different authors proposals on the elements contained in the same. We analyze its consistency according to what we mean by community social praxis. Also, we wonder about the role of certain entities that may be involved in the work of a genuine praxis. We note the relationship between the position of the community mediators in relation to the ideas that can be built around the concept of community. Finally, we required that from any discipline of social studies, including the SCP, it requires work on the reprocessing of those preconceived certainties to avoid falling into asymmetrical power relations with participants in community projects.
\end{abstract}

Keywords: Social community Psychology (SCP), social praxis, community social praxis, community.

Pareciera difícil encontrar un punto de equilibrio entre la postura de un investigador y su campo de interés, debido a que sus propios prejuicios o la fantasía de querer tener un control absoluto pueden volverse su peor enemigo. A estas cuestiones nos enfrentamos cuando queremos darle sentido a la realidad con base en ciertas concepciones que pueden estar distantes o ser ajenas a tal fin. Por lo tanto, cuestionar nuestras certidumbres es el mejor antídoto para cualquier enfermedad enciclopédica.

En este trabajo cuestionamos la certeza que puede prevalecer en torno al uso del concepto comunidad desde la Psicología Social Comunitaria (PSC) ${ }^{1}$, al analizar algunas implicaciones teórico-conceptuales en la reelaboración del término, ya que aun cuando se considera esencia de la PSC, su análisis resulta paradójicamente austero (Krause, como se citó en Alfaro \& Berroeta, 2007) porque "es poco frecuente encontrar en las publicaciones aproximaciones conceptuales al término, y cuando lo hacen, presentan un desarrollo limitado" (Zúñiga, como se citó en Tovar \& Ángeles, 2004, p. 28). Esta flexibilidad ha generado una diversidad de conceptualizaciones que no siempre son congruentes entre sí, por lo que el término ha sido catalogado como un misterio incomprensible
(Guareschi, como se citó en Saforcada \& Castellá, 2008) o mágico en el que pareciera que "todo se vale".

Esta apertura equivaldría a ver un árbol que va creciendo y da frutos pero del cual no observamos con claridad las raíces que lo mantienen firme. Los seres humanos tendemos a dar por sentado ciertos significados sin corroborar a cuál término se refiere; de ahí que sea común usar a priori las siguientes afirmaciones: "como se sabe", "como se entiende". Aseveraciones que se han desplazado hacia al campo del saber académico-científico, ocasionando que se empleen conceptos con diversos niveles de desconocimiento. Asoma una especie de miedo

1 Desde algunos autores latinoamericanos como Montero (2003) y Baró (1990) entre otros, la Psicología Comunitaria busca "...el desarrollo del control y el poder de los actores sociales comprendidos en un proceso de transformación social y psicosocial que los capacita para realizar cambios en su entorno y a la larga en la estructura social" (Montero, 2003, p.43). Obedecen a la postura latinoamericana que también toma en cuenta algunos de los postulados de la Teología de la Liberación en coincidencia con la metodología participativa, la búsqueda de la justicia y la solidaridad como auto-investigación y auto-gestión (Jiménez, 2004). 
a equivocarnos, motivado por la adherencia a determinadas visiones positivistas. Por lo tanto, en esta serie de reflexiones nos permitimos equivocarnos, sin temer a lo extraño (Sawai, como se citó en Alfaro \& Berroeta, 2007), para observar la riqueza del concepto en un proceso de construcción permanente, nunca acabado.

¿Para qué es necesario cuestionar los conceptos dentro de una ciencia? Para visualizar si hay una sola voz como canto de sirenas o para reconocer que es la comunidad misma como realidad la que se impone diversa, caótica y heterodoxa. Esta postura nos conduce a asumir, axiológicamente, una forma distinta de mirar la praxis en comunidad (Ornelas, 2008) y de facilitarnos un diálogo interior para observar nuestra manera de percibir la realidad y cuestionarla (Foladori, como se citó en Alfaro \& Berroeta, 2007).

Un primer punto que puede cuestionarse es aquel que se refiere al uso del término en el campo de la piscología. Al consultar determinadas referencias que aluden a él (Tovar \& Ángeles, 2001), nos damos cuenta que se encuentra desarticulado de la visión que promueve la PSC. Esta situación conceptual nos ofrece una explicación para reconocer las primeras provisiones bibliográficas al margen de la realidad latinoamericana, procedentes de países anglosajones o de norteamericanos donde su historicidad dista de las grandes necesidades sociales, de las luchas y de la esperanza de la sociedad latinoamericana.

En el caso del ámbito anglosajón, los términos de "psicología comunitaria y salud mental comunitaria se usan como términos similares" (Musitu, 2004, p.6). En Estados Unidos, en una revisión hemerográfica realizada de 1973 a 1982 , se ha constatado que predominaban los estudios centrados en la salud mental. En los 80 , a pesar del ajuste psicosocial del sujeto, se continuó ligando la idea de salud mental que se reflejaba en las intervenciones preventivas dirigidas al desarrollo de competencias personales. En otros contextos como Reino Unido, el desarrollo de la PC es bastante reciente y la literatura está elaborada por psicólogos con un enfoque más clínico y tradicional (Gesten \& Jason, como se citó en Musitu, 2004).

Nuestras reflexiones nos llevan a cuestionarnos acerca de cómo se relacionan los planteamientos teórico-conceptuales con la reelaboración que pretendemos hacer del término comunidad. Pareciera que la PSC, en su propio proceso de construcción y reconstrucción disciplinar, recurrió inicialmente a conceptos que se encontraban en la vertiente positivista como única opción. Complicidad denunciada por Baró (1990), en cuanto a que apuntaba hacia la intensión encubridora de los métodos positivistas, afincados en el corazón mismo de la psicología del primer mundo, aquella que escondía, bajo capa de imparcialidad y objetividad, el ejercicio del poder por medio del cual una minoría determinaba el tipo de problemas sobre los que debían interesarse los psicólogos.

La organización de la ciencia, en lugar de un ejercicio de servicio, se convertía en uno de poder, que al ser indagado encontramos que florece en el "yo" quien por instaurar su discurso establece una mirada hegemónica ante los demás, consciente algunas veces e inconsciente otras, desde la fantasía del sistema imperante; es decir; una postura de experto ante un enfermo. Adentrémonos, entonces, en un análisis más pormenorizado del manejo que han realizado algunos autores en torno a aspectos centrales en el concepto de comunidad.

Al observar las propuestas de Guareschi (como se citó en Saforcada \& Castellá, 2008), Krause (como se citó en Alfaro \& Berroeta, 2007), Tovar \& Ángeles (2001) y Heller (como se citó en Velarde, 2006), acerca de los elementos que contiene el término comunidad, encontramos que algunos de ellos difieren de los principios asumidos por la PSC con relación a lo comunitario y que, por el contrario, se asemejan a la tendencia de mantener el control de las ideas desde la ciencia. Al respecto, Guareschi (como se citó en Saforcada \& Castellá, 2008), 
retomando la "Visión del eje", "Ser humano" y del eje "social", en relación a lo individual dentro del concepto de comunidad, los observa como elementos opuestos, no dinamizantes entre sí ni entre los demás. Tal postulado llevaría a visualizar a la comunidad como algo estático, que se perpetúa; es decir, una concepción incompleta que subestimaría la relación entre hombre y sociedad.

En un segundo aspecto a revisar, la "delimitación territorial" se refiere a la definición de comunidad cuando se alude a ésta en relación a un lugar. Lo subjetivo del asunto es que en la mayoría de los casos, los límites se fijan por criterios político-administrativos y en algunos otros por el azar, dejándose de lado los agrupamientos y las tramas de interacción que ciertamente son significativas para la configuración de lo comunitario. Observar a la comunidad por los límites físicos puede conducir sutilmente a una "marginación, a una participación acotada...." Por lo que, si la PSC busca ser liberadora de las formas de comprender lo comunitario, el elemento territorial o físico no resulta correspondiente, pues forma parte de "los criterios simplistas de carácter administrativo-político tampoco suficientes a la hora de estudiar e implementar políticas sociales para estructuras sociales comunitarias" (Diéguez, 2000, p. 15).

La noción de territorio se desvanece pues, actualmente, se establecen redes y agrupaciones de personas que no comparten una ubicación geográfica común y que ni siquiera mantienen contacto cara a cara (Krause, como se citó en Alfaro \& Berroeta, 2007); es decir, no se conocen de manera personal, pero pueden estar formando parte de una comunidad. Consideramos, por lo tanto, que la noción de territorio como espacialidad física no debiera considerarse de forma central en la reelaboración

2 El autor realza las consecuencias de enfocarse sólo en la visión del ser humano o sólo de la social, pues se perdería su relación. del concepto de comunidad; tampoco, como ya se comentó, que ciertos sectores poblacionales sean delimitados por determinados programas gubernamentales como comunidades, ya que observamos cómo algunos de estos programas de carácter social se dirigen a la comunidad designándola a priori como "comunidad urbano-marginal." Esta forma de mapeo se relaciona cuando se intenta definir un sector geográfico, caracterizándolo por la miseria derivada del anárquico y desigual crecimiento urbano, en una especie de rótulo que la aísla de su propia dinámica para justificar la ayuda externa (Jiménez, 2004, p. 136).

Folarodi (como se citó en Alfaro \& Berroeta, 2007), señala que tal ayuda se estructura siempre en un cierto "desnivel", por lo tanto, el necesitado queda en deuda, anclado con el proveedor que da inicio a una dependencia - ejercicio de asistencia. Según el autor, antropológicamente, se ha demostrado que en muchos pueblos no existe el regalo. Si se recibe algo hay que dar algo a cambio para evitar quedar en deuda. Asumir esta postura es interrogarnos acerca de cómo se ha ido catalogando la idea de comunidad desde el origen de la PC. Como lo hemos mencionado líneas arriba, en E.U y otros países anglosajones, lo territorial, así como lo urbano parecieran haber tenido un desplazamiento hacia la visión diagnóstica de la salud mental desde la cual se le impide a la comunidad tener voz propia.

Un tercer aspecto que deseamos cuestionar es el de "la idealización a priori de la realidad" que se convierte en un punto de crítica, al mezclar el "deber ser" idealizado de las comunidades con el concepto mismo desde su sentido teórico tradicional; es decir, aquel que apunta a una visión funcional-positivista. Tal situación es preocupante, pues el problema yace en que este componente de valoración de una "comunidad ideal" pierde su objetividad como herramienta teórica para delimitar el objeto de estudio de la intervención (Krause, como se citó en Alfaro \& Berroeta, 2007), con énfasis en 
la prevención que tiende a confundirse con la ausencia de conflicto (Jiménez, 2004).

Esta concepción quebranta el reconocimiento del conflicto como un recurso comunitario; pareciera asumirse desde su ausencia para el trabajo en comunidad, olvidando que hablamos de seres humanos cuya naturaleza es imperfecta, conflictiva y por tanto contradictoria. Lo que resulta inaceptable en posturas asépticas que buscan un ideal inalcanzable e inducen a que las personas se frustren.

Por otro lado, hace falta analizar también la creencia de que la comunidad se puede identificar a través de una dimensión temporal. Hoy, más que en otro tiempo, las redes sociales, los medios masivos de comunicación y las nuevas tecnologías nos obligan a comprender la dimensión de la temporalidad y la espacialidad de forma flexible (Krause, como se citó en Alfaro \& Berroeta, 2007), agregaríamos, polisémica, abierta, diversa. La tendencia actual muestra que la pertenencia a una comunidad no es necesariamente de por vida, puesto que ésta puede ser reducida y exclusiva o no. En esta dimensión de temporalidad podemos observar nuevamente una tendencia reduccionista al momento de concebir a la comunidad, pues pareciera que lo necesario radica en la delimitación de la interacción humana en un solo tiempo y en un solo espacio, misma que va más allá de lo que se pueda analizar.

De ahí que estemos en contra de la idea funcional-positivista que obliga a hablar de la comunidad como algo acabado y concreto; el desencuentro es evidente con la propuesta emergente que asumimos desde la PSC, ya que esta última nos traslada a la búsqueda de una re-conceptualización desde los propios actores de la comunidad, en congruencia con el objeto de su abordaje psicosocial que busca "el desarrollo del control y el poder de los actores sociales comprendidos en un proceso de transformación social y psicosocial que los capacita para realizar cambios en su entorno y a la larga en la estructura social" (Montero, 2003, p. 143).
Fomentar o potenciar la participación de la comunidad en la elaboración de un quehacer comunitario propio que permite que la capacidad de reflexión y de creación salga a la luz ${ }^{3}$ para reconocer la fortaleza de los actores que la definen y conducirnos al reconocimiento de la sociedad como una construcción colectiva, a su vez, influida y susceptible de crítica y cambios (Montero, como se citó en Jiménez, 2004). La elaboración del propio concepto de comunidad es una escala de acciones futuras que puede desarrollarse propiciando el diálogo de los actores sociales, capaces de monitorear las fortalezas y oportunidades que pueden mejorar en su propio contexto para proponer y hacer los cambios requeridos, en contraposición con el concepto ideal de comunidad, donde todos los componentes y los procesos estarían acabados.

Es prudente apuntar la necesidad de crear una PSC coincidente con el desarrollo que demande la comunidad (Montero, 2003). En ello hay una exigencia por demostrar, a partir de acciones simples o de mayor complejidad, y donde quienes trabajan en calidad de facilitadores(as) comunitarios reconozcan el lugar que ocupan los propios participantes para que sean ellos(as) quienes valoren y definan su sentido de ser y pertenencia a la comunidad. Se trataría de evitar la mirada academicista que suele empatar con concepciones ya definidas o cosificantes.

Por lo tanto, en este trabajo pretendemos poner de manifiesto lo siguiente como primera premisa: hay que realizar un acercamiento con la comunidad para elaborar una noción propia del contexto a partir de las características observadas y de las experiencias vividas

3 Decimos que se exteriorice porque no cabe duda que hay una concepción por parte de quienes habitan en la comunidad y puesto que la construcción de una imagen colectiva del lugar que nos es propio responde a un proceso colectivo en tanto se internaliza como un proceso de orden social. 
por quienes la habitan. De esa forma, situamos el ejercicio de la praxis comunitaria desde una postura que resulta abierta y en constante construcción, incluyendo la meta a la cual estén dispuestos a llegar los involucrados. Ello sugeriría considerar los elementos ideales no desde el ámbito teórico-profesional, sino desde la cosmovisión de los integrantes del espacio en construcción. Sin duda, el papel de las y los facilitadores comunitarios(as) es únicamente de carácter mediador; en tal sentido podemos nombrar a este proceso conjunto como "real" y no como "ideal".

Asimismo, en función de otra premisa, afirmamos la necesidad de elaborar una conceptualización derivada de las situaciones reales o vividas con la perspectiva de la Psicología Social Latinoamericana descrita sucintamente. Ejercicio que representaría un concepto de comunidad útil como herramienta teórica-empírica para observar al objeto de estudio en el trabajo comunitario como un estado en permanente cuestionamiento (Krause, como se citó en Alfaro \& Berroeta, 2007).

Por lo tanto, proponemos como momento de integración la visión del eje individual y social de forma relacional, puesto que no existe el individuo aislado ni el grupo-máquina cerrado, sino seres humanos en constante construcción y reconstrucción (Guareschi, citado por Saforcada \& Castellá, 2008). Dicha postura facilita, por otra parte, la visión de la comunidad a través de algunas perspectivas como la propia propuesta ecológico-sistémica (Bronfenbrenner, como se citó en Corsi, 1997), que permite su análisis colectivo más amplio.

Estamos de acuerdo en tomar en cuenta el sentido que adquieren las prácticas de la vida cotidiana (Heller, como se citó en Velarde, 2006), como formas de expresión simbólicas que representan un vehículo para comprender la "trama relacional" de los diferentes actores e instituciones en la conformación de la comunidad. Esta postura nos permite entender que las comunidades asumen "configuraciones subjeti- vas" diversas, pues su universo simbólico deviene tanto de lo individual como de lo colectivo acontecido. Tal concepción implica que no podemos realizar generalizaciones de las comunidades (Tovar \& Ángeles, 2001); es decir, hay que hablar de diversidad de comunidades. Verlas en su expresión cotidiana, sin duda, confirma que no hay ni temporalidad ni espacialidad fijas, en un ejercicio que busca su transformación de lo heterónomo a lo autónomo.

Hasta ahora, planteamos dos caminos susceptibles de considerar en la concepción de una comunidad. Del mismo modo, alertamos sobre el uso de un lenguaje prejuicioso que suele estereotipar a algunas comunidades como "urbano-marginales", "delictivas", "pobres" "pandilleras", entre otras formas. Sobra decirlo, estas denominaciones resultan en generalizaciones peyorativas que incitan al aislamiento comunitario, al pretender justificar la ayuda externa de manera semejante a la actuación de los hospitales psiquiátricos, donde el "loco" es el que necesita la ayuda, desplazando la visión comunitaria holística, al seguir favoreciendo el control mediante una participación acotada (Diéguez, 2000).

En el caso concreto de ciertos programas y proyectos gubernamentales, sugerimos evitar las visiones unidireccionales que intentan definir la forma de asumir a la comunidad a partir de racionalidades centradas en indicadores cuantitativos y, en el peor de los casos, de orden ideológico-político, nada cercanas a las necesidades de los beneficiarios de esos programas o proyectos comunitarios.

Esbozada la propuesta, arribamos al recuento de esta serie de ideas que nos lleva a otras interrogantes. Nuestras observaciones parten de una crítica propositiva que rechaza dar por sentada una comprensión acabada de lo que debemos entender por comunidad. Postura que nos lleva a reflexionar, en el campo de las ciencias sociales, sobre el modo de concebir los sustentos epistemológicos y teóricoconceptuales, con base en un cuestionamiento 
permanente, no solo receptivo, sino también crítico-constructivo. Por ello, resulta pertinente cuestionar la postura que se asume en el proceso de formación universitaria de nuevos profesionales en el campo de la PSC, a partir de un marco pedagógico que nos lleva a interrogarnos: ¿se propicia el debate de posturas teóricas diversas?

En el mismo sentido, la elaboración de planes de estudio y los diseños curriculares implicarían una nueva interrogante: ¿son estos planes y programas pensados o elaborados para cumplir un requisito académico o con el objetivo de buscar nuevas formas de acercamiento para la comprensión y diferenciación de posturas diversas? Significa la oportunidad de cuestionar la certidumbre e inspeccionar lo que nos motiva en nuestro quehacer, sea este académico, o laboral-profesional. Lo anterior supone fomentar un análisis teórico para que los y las futuros(as) profesionales tengan la posibilidad de comprender la importancia que adquiere andar un trayecto académico congruente, es decir, desde una postura epistemológica que posibilite aclarar los sustentos. De esta forma, podemos evitar la yuxtaposición de corrientes teóricas y autorales que no coincidan en principios. Estas son algunas de las ventajas de promover el gusto por la investigación y es nuestra responsabilidad cuestionar(nos) para acercarnos a reflexiones teórico-conceptuales y metodológicas más claras y flexibles que, sin duda, nunca serán perfectas.

Consideramos que, en ocasiones, nos olvidamos concebir la investigación documental como proceso más que como producto, de ahí la necesidad de poner en duda la terminología que se emplea para encontrar conceptos afines. Es fundamental observar cómo según la corriente donde se extrae el concepto, se marcan diferencias epistemológicas que, sin duda, determinan el camino de una trayectoria investigativa.

Una actitud que facilitará aceptar la apertura a la duda y al cambio de lo establecido en el manejo terminológico es la humildad que permita observar la reconstrucción conceptual como un proceso histórico y de mejora en la investigación. Ruta sugerida para incursionar en este proceso, con la idea de que la memoria histórica siempre será susceptible de modificaciones pues somos seres dialécticos, en construcción permanente, y por lo tanto todo lo que creamos también lo será. El conocimiento es un proceso infinito destinado a mejorar continuamente (Montero, como se citó en Alfaro \& Berroeta, 2007). Es necesario asumir la responsabilidad de quienes somos formadores académicos y, desde el ámbito en donde nos desarrollemos, comprender la magnitud del papel que desempeñamos. Significa iniciar un proceso de humanización, en donde empecemos a librarnos poco a poco de las fuerzas sociales y de experiencias previas que nos hacen concebir a los involucrados en la investigación como objetos de estudio y no como seres humanos (Freire 1970, como se citó en Saforcada \& Castellá 2008).

Como vemos, este trabajo no solo propicia la reflexión en el campo de la PSC, sino en toda disciplina social. Si retomamos la importancia que adquiere la reelaboración del concepto de comunidad, esta postura nos permite percibir comunidades donde muchos sólo ven gente con problemas. Es una oportunidad para evitar trampas que parecieran provenir de discursos de tipo diagnóstico que suponen al otro en desventaja. Desde cualquier óptica disciplinar valdría la pena prescindir de terminología a priori que nos lleve a la desilusión de una realidad antes de conocerla. Es necesario realizar un trabajo de campo más que de escritorio para desvestirnos de prejuicios sujetos al ego de "saberlo todo". En ese sentido, la Investigación Acción Participativa (IAP) 4 (Balcázar, 2003; Montenegro, 2004) apunta a descobijarnos

4 Es por ello que se dice, la IAP no es sólo un método de investigación, sino un estilo de vida. 
de la comodidad del investigador de gabinete para llevarnos a encontrar en los miembros de la misma a personas que nos pueden mostrar su experiencia de vida hasta convertirla en un proceso mutuamente compartido.

Por otra parte, se recomienda analizar las condiciones contextuales e institucionales de los espacios donde se realiza la práctica profesional, para observar los elementos estructurales y sus intereses. De esa forma, la diferenciación de las características favorecedoras del trabajo en conjunto es factible. Este ejercicio diferenciador nos ha de conducir a la construcción de lo que entendemos por "praxis" en la PSC y aprender a capitalizar los recursos tanto propios como de la comunidad para propiciar y fortalecer su crecimiento, así como el proceso de negociación de aquellas condiciones que dificulten el encuentro. El propósito es prever la pérdida de identidades comunitarias frente a programas o proyectos de corte utilitario.

Acentuamos que la idea de individuo como elemento central del abordaje en la praxis se aleja del trabajo comunitario; algunas ciencias han observado entre sí su objeto de estudio en un sentido individualista, que define posturas competitivas, motivadas por el pensamiento de "sin competencia no hay progreso". (Guareschi, como se citó en Saforcada \& Castellá, 2008). Ponemos sobre la mesa de discusión la necesidad de cambiar estas posturas para comprender la urgencia de la interdisciplinariedad con el fin de romper los esquemas del temor al cambio: de enseñar a ser aprendices. Manifestamos nuestro desacuerdo cuando en el discurso de una disciplina se asumen posturas de superioridad ante otras que obstaculizan el encuentro. Dudar de las tendencias positivistas y elitistas nos acerca a un discurso transdisciplinar de lo que significaría una comunidad.

Desde la experiencia de quienes escribimos estas reflexiones, el ejercicio de una praxis cobijada por la PSC recién ha comenzado; es ampliamente recomendable llevar a cabo una labor de recopilación constante que reúna la producción investigativa dé cuenta de un estado en desarrollo. El escenario desde el cual participamos y desarrollamos un proyecto de investigación e intervención sociocultural es el de la Facultad de Psicología y Terapia de la Comunicación Humana de la Universidad Juárez de Durango, (Guzmán, Ortiz, \& Alquisiras, 2012; Guzmán, Ortiz, Alquisiras, \& Barragán, 2010). Hemos podido desarrollar un diálogo constante entre diversos momentos o etapas del proceso de investigación aplicada que intentamos realizar, como un ejercicio de praxis comunitaria, con el objetivo de encontrar hallazgos en un proceso de construcción compartido, que nos ha permitido ser lectores/as de algunas premisas que los y las propios/as compañeros/as facilitadores del trabajo en comunidad nos han ofrecido. De esta forma, dicha experiencia está destinada a capitalizar la mirada de otros/as en las propias de los y las psicólogos/as en formación, de pasantes y egresados de la licenciatura, así como de profesores/as de la facultad interesados/as en el área. Aunado a lo anterior, participamos de procesos permanentes de formación en PSC y otras disciplinas sociales orientadoras de nuestro ejercicio de praxis en las comunidades donde realizamos trabajo de campo.

La relación entre un proyecto, un proceso de formación y la praxis social permanente son aportes indispensables para fortalecer la experiencia empírica que cobija el análisis de estas reflexiones, más allá de su carácter conceptual. Nuestra experiencia ha sido producto del desarrollo y el encuentro a partir de talleres psicoeducativos y culturales comunitarios que llevamos a cabo desde hace siete años. De nuevo más preguntas ¿qué va primero, la vida cotidiana, la teoría, el ser humano o el ejercicio profesional?

Partir de un plan completo o en transformación durante el ejercicio de praxis, nos ha permitido vislumbrar la redimencionalización de las relaciones de poder entre el saber académico y el conocimiento popular. Es un cambio de 
significados y sentidos en las relaciones, pues nos concentramos en un compartir con los y las otro(as), independientemente de la clase, el género, la etnia, la generación, el idioma o el conocimiento adquirido. Tal práctica nos ha guiado hacia la identificación de momentos donde parecen desvanecerse esas relaciones impuestas y emerger toques de nuevas relaciones entre seres humanos. ¿Quién dijo que investigar significa posicionarse sobre otros? ¿Acaso investigar implica compartir para enriquecer las perspectivas de vida como una creación conjunta? Es aquí donde resaltamos la postura genuina que nos lleve a meditar, desde nuestro campo académico y en el proceso de observación, sobre un nuevo camino para hacer investigación aplicada con base en una nueva conceptualización de comunidad, aquella que vislumbre respuestas a nuevas interrogantes y dé lugar a mejorar como seres humanos.

Finalizamos este trabajo con la idea de contribuir en la generación de una reflexión con el objetivo de avanzar en las revisiones teóricoconceptuales de la PSC y otras disciplinas que pueden tomar como ejemplo este ejercicio favorecedor de procesos de diálogo continuos, de análisis y discusión acerca del papel que juegan hoy día los profesionales comunitarios en su esfuerzo por construir una praxis académica en comunión con una praxis comunitaria.
Alfaro, J., \& Berroeta, H. (2007). Trayectoria de la Psicología Comunitaria en Chile prácticas y conceptos. Chile: Universidad de Valparaíso.

Balcázar, F. (2003). La investigación-acción participativa en psicología comunitaria. Principios y retos. Apuntes de psicología, XX 1 (3), 419435.

Baró, M. (1990). Psicología de la Liberación para América Latina. Salvador: UCA Editores.

Corsi, J. (1997). Violencia Familiar: una mirada interdisciplinaria sobre un grave problema social. Buenos Aires: Altamira.

Diéguez, A. J. (2000). La intervención Comunitaria -experiencias y reflexiones. México: Espacio Editorial.

Guzmán, M. R., Ortiz, M.L., \& Alquisiras, L. (2012). Significación y Realidad: una propuesta de intervención comunitaria integradora. En I. Serrano García (Comp.), Aproximaciones a los problemas sociales contemporáneos. Volumen II (pp.43-54). Puebla: Universidad Iberoamericana.

Guzmán M. R., Ortiz M.L., Alquisiras, L., \& Barragán L.E. (2010). La experiencia de una intervención psicosocial comunitaria desde la academia, la práctica en campo y la auto reflexión. Revista Enlaces académicos, 3(2), 93-103.

Jiménez, I. (2004). La Psicología Social Comunitaria en América Latina como Psicología Social Crítica. Revista de Psicología de la Universidad de Chile, 1, 133-142.

Montenegro, M. (2004). Investigación Acción Participativa. En G. Musitu, J. Herrero, L. Cantera, \& M. Montenegro, Introducción a la Psicología Comunitaria, (pp. 135-161). Barcelona: Ed. UCO

Montero, M. (2003). Teoría y Práctica de la Psicología Social Comunitaria, la tensión entre comunidad y sociedad. Buenos Aires: PAIDOS.

Musitu, G. (2004). Introducción a la psicología comunitaria. Barcelona: UOC.

Ornelas, J. (2008). Psicología Comunitaria. Lisboa: FIM DE SÉCULO.

Sacristán, G. J., \& Pérez, G. I. (1992). Comprender y transformar la enseñanza. El currículum: ¿Los contenidos de la enseñanza o un análisis de la práctica? Madrid: Morata.

Saforcada, E., \& Castellá, S. (2008). Enfoques Conceptuales y Técnicos en Psicología Social Comunitaria. Buenos Aires: PAIDOS.

Tovar, P., \& Ángeles, M. (2001). Psicología Social Comunitaria: Una alternativa teórico metodológica. Barcelona: Plaza y Valdés.

Velarde, S. (2006, 04 de abril). Sociolog ía de la Vida Cotidiana. Ponencia presentada en el ciclo de temáticas, problemáticas en Sociología, Universidad Autónoma de Ciudad Juárez. 